\title{
Oxide Evolution During the Solidification of 316L Stainless Steel from Additive Manufacturing Powders with Different Oxygen Contents
}

\begin{abstract}
XINLIANG YANG, FENGZAI TANG, XINJIANG HAO, and ZUSHU LI
The oxide evolution during the solidification of $316 \mathrm{~L}$ stainless steel from additive manufacturing powders with different oxygen contents is studied by in situ observation of the melting and solidification of the powder materials, advanced characterization of the solidified materials, and non-equilibrium thermodynamic analysis. An oxide evolution map is established for the $316 \mathrm{~L}$ powders with different oxygen contents. It reveals the relationship between the surface oxidation in the reused powder and its expected oxide species and morphology in the as-solidified component. For the $316 \mathrm{~L}$ powder with oxygen content higher than $\sim 0.039$ pct, the liquid oxide formed first from the steel melt and then crystallized to certain oxide phases during solidification, while for the powder with lower oxygen, oxide phases are suggested to directly form from the steel melt. The oxide species in the as-solidified sample was predicted by the Scheil-Gulliver cooling calculation and verified by the TEM-based phase identification. The oxides formed in the melt of low $\mathrm{O} 316 \mathrm{~L}$ alloy $(0.0355$ pct $\mathrm{O})$ are predicted to be $(\mathrm{Mn}, \mathrm{Cr}) \mathrm{Cr}_{2} \mathrm{O}_{4}$ spinel and $\mathrm{SiO}_{2}$ oxide. In the high $\mathrm{O}(0.4814$ pet $\mathrm{O}) 316 \mathrm{~L}$ melt solidification, the final oxides formed are $(\mathrm{Mn}, \mathrm{Cr}) \mathrm{Cr}_{2} \mathrm{O}_{4}$ spinel, $\mathrm{SiO}_{2}$ oxide, and $\mathrm{Cr}_{2} \mathrm{O}_{3}$ corundum. As an important characteristic of powder materials, the oxygen pick-up due to the powder surface oxidation significantly influences the inclusion evolution in the powder fusion process.
\end{abstract}

https://doi.org/10.1007/s11663-021-02191-w

(C) The Author(s) 2021

\section{INTRODUCTION}

THE research of the inclusion evolution is one of the critical considerations in the steel metallurgy, which has extensively focused on the reaction between the intrinsic elements (e.g., $\mathrm{O}, \mathrm{N}, \mathrm{H}, \mathrm{C})$ and the metallic elements, and the sulfides and phosphide impurities in the initial steelmaking and subsequent thermomechanical processes. $^{[1]}$ By the extended application of the powder formed steel materials via the powder fusion (liquid-solid transition)-based process, ${ }^{[2]}$ the fundamental understanding of inclusion formation and evolution was demanded due to its non-equilibrium nature ${ }^{[3]}$ for the powder manufacturing and additive manufacturing (AM) technology marching into the structure component business. ${ }^{[-6]}$

XINLIANG YANG, FENGZAI TANG, and ZUSHU LI are with the WMG, University of Warwick, Coventry CV4 7AL, UK. Contact e-mail: Z.Li.19@warwick.ac.uk XINJIANG HAO is with the Liberty Powder Metals, Materials Processing Institute, Eston Road, Middlesbrough, TS6 6US, UK.

Manuscript submitted January 4, 2021; accepted April 14, 2021.

Article published online May 10, 2021.
The inclusion evolution under the non-equilibrium state has been addressed in the steel welding research $;{ }^{[7-10]}$ however, due to the inherent feedstock difference, the inclusion/oxide systems between the steel welding and the powder-formed steel fusion are less comparable. The early study of the inclusion evolution in powder fusion has focused on the processing aspect. Song et al. ${ }^{[11]}$ have emphasized the importance of the protective gas purity on the oxide inclusion formation and reported that varying oxygen contents in the atmosphere of the laser solid forming process significantly changed the morphology and amount of the oxide inclusion in the as-processed samples. Eo et al. ${ }^{[12]}$ studied the effect of processing parameters (laser power, scan speed, beam spot size and powder steel composition) on the size distribution and number density of the inclusion evolution in the laser powder deposition process and proposed a linear relationship between the inclusion number density and the tensile yield strength of the as-processed components. Recently, Deng et al. ${ }^{[13]}$ reported a wide range of experimental results of the oxygen source on the formation of the oxide inclusion from the precursor powder, processing atmosphere to the moisture during the powder storage. The abovementioned investigations nicely covered the processing 
aspect of inclusion evolution, but limited information on the oxide evolution during the powder fusion process was reported from the material quality point of view.

With the rapid development of additive manufacturing technology, the significance of extending the powder service life to improve the process cost-effectiveness has drawn great attention. ${ }^{[14,15]}$ Researchers have studied the effect of the reused powder on the characteristics of powder flowability, packing density ${ }^{[16,17]}$ and resultant mechanical performance of the as-processed components. ${ }^{[18-20]}$ However, the effect of the reused powder with different oxygen pick-up contents on the oxide formation and evolution in the powder fusion is still not clear.

Therefore, this study aims to reveal the oxide formation and evolution in the non-equilibrium solidification of the $316 \mathrm{~L}$ stainless steel from the additive manufacturing powders with different surface oxidation conditions. The steel powders with different oxygen contents were subjected to the in situ observation of melting and solidification under the high-temperature confocal laser scanning microscope (HT-CLSM), TEM-based phase identification and non-equilibrium thermodynamic analysis. A relationship between the powder oxygen pick-up and the oxide formation route during the solidification is proposed. The solidified oxide species from different powder conditions were analyzed using the Scheil-Gulliver cooling function and confirmed by the crystallographic identification.

\section{MATERIALS AND METHODS}

\section{A. The 316L Powder Materials Under Different Oxygen Pick-up Conditions}

Three 316L powders with different oxygen contents (Table I), i.e., low $\mathrm{O}$ powder, medium $\mathrm{O}$ powder and high $\mathrm{O}$ powder were used in this study, representing three oxygen pick-up conditions of the steel powders in their service life in the powder fusion-based AM process. The low $\mathrm{O}$ powder was the as-received $316 \mathrm{~L}$ powder produced by inert gas atomization with limited oxygen pick-up, and the medium $\mathrm{O}$ powder was the rejected powder in the powder fusion process of the as-received $316 \mathrm{~L}$ powder. The high $\mathrm{O}$ powder representing excessive-high oxygen pick-up in process was produced by oxidizing the as-received $316 \mathrm{~L}$ powder at $800{ }^{\circ} \mathrm{C}$ under compressed air with $0.2 \mathrm{~L} / \mathrm{min}$ flow rate for 20 minutes to simulate the oxygen pick-up level reported in the research community. ${ }^{[19,21]}$

The chemical compositions of the three types of powder with different surface oxidation conditions are listed in Table I. The chemical composition of metallic elements was determined by the inductively coupled plasma mass spectrometry (ICP-MS) method. Carbon, sulfur and phosphorus contents were obtained by combustion analysis. Oxygen content was measured by inert gas fusion. Morphology of these powders is shown in Figure 1 with the feature of the small powders adhering over large ones, which is commonly seen in powder manufacturing due to the impingement of solidified fine powders over the coarse molten/semimolten powders in the powder atomization process. ${ }^{[24]}$ A clean surface is observed in the low $\mathrm{O}$ powders in Figures 1(a) and (d). The oxygen pick-up in the Medium $\mathrm{O}$ powder led to the contamination formed on the powder surface (Figure 1(e)), while the excessive oxygen pick-up in the high $\mathrm{O}$ powder resulted in the surface oxides covering the individual steel powders in Figures 1(c) and (f). According to the previous studies, ${ }^{[15,25-28]}$ the $\mathrm{Si}, \mathrm{Mn}$ and $\mathrm{Cr}$, as oxygen getter elements in the $316 \mathrm{~L}$ stainless steel, preferentially participated in the powder surface oxidation. With further extended oxidation conditions, $\mathrm{Fe}$ could also be involved in forming iron oxides.

\section{B. Characterization of Powders and Solidified Droplets}

To study the effect of $316 \mathrm{~L}$ powder with different oxygen pick-up contents on the inclusion evolution during the powder fusion, the Yonekura VL2000DX-SVF17SP high-temperature confocal laser scanning microscope (HT-CLSM) was utilized to in situ observe the oxide/metal interaction with higher morphological contrast comparing to high energy synchrotron X-ray approach. ${ }^{[21]}$ This can simulate a non-equilibrium solidification condition which can be captured by the laser optical system using suitable cooling rate, and exclude the local ionization and powder spatting due to the complicated physical phenomenon of laser beam/powder interaction. ${ }^{29]}$ The powder sample was heated to $1600{ }^{\circ} \mathrm{C}$ at a heating rate of $10 \mathrm{~K} / \mathrm{s}$. After 20 seconds holding at $1600{ }^{\circ} \mathrm{C}$, the melt was then rapidly solidified at the cooling rate of $10 \mathrm{~K} / \mathrm{s}$ in a non-equilibrium state. The Ar atmosphere (N5.0, BOC) at the flow rate of $0.2 \mathrm{~L} / \mathrm{min}$ was applied in the programed thermo-cycles. $0.25 \mathrm{~g}$ powder dose was used to simulate the interaction volume of a single beam spot with powder material without the interference of the powder spreadability. ${ }^{[30]}$ For each powder conditions, seven sets of thermo-cycle were conducted for in situ observation and as-solidified droplet preparation.

The post-mortem characterization of the formed oxide phase in the as-solidified droplet was conducted by the morphological, chemical and crystallographic analysis. A scanning electron microscope (SEM, JEOL $7800 \mathrm{~F}$ ) and a dual-beam focused ion beam (FIB) SEM (Versa FEI, now part of Thermo Fisher Scientific) were employed to study the surface morphology of the as-solidified droplet, during which both secondary electron (SE) and backscattered electron (BSE) images were acquired. Chemical compositions of the samples were examined using the equipped energy-dispersive X-ray spectroscopy (EDS, Oxford Instruments) at 10 to $15 \mathrm{kV}$. Cross-sectional TEM (transmission electron microscopy) samples were sectioned from the oxide layer of the as-solidified 316L droplet using the standard in situ lift-out technique. Scanning TEM (STEM) analysis was conducted using a Talos F200X microscope (FEI, now part of Thermo Fisher Scientific) operating at 200 kV with a Super-X EDS system. 
Table I. The Chemical Compositions (Wt Pct) of Three 316L Powders With Different Oxygen Pick-up Conditions

\begin{tabular}{|c|c|c|c|c|c|c|c|c|c|c|}
\hline & $\mathrm{Cr}$ & $\mathrm{Ni}$ & Mo & $\mathrm{Mn}$ & $\mathrm{Si}$ & $\mathrm{C}$ & $\mathrm{S}$ & $\mathrm{P}$ & $\mathrm{O}$ & $\mathrm{Fe}$ \\
\hline Low O & 15.77 & 10.22 & 2.12 & 1.26 & 0.63 & 0.0147 & 0.0142 & 0.038 & 0.0355 & bal. \\
\hline Medium $\mathrm{O}$ & 15.74 & 10.23 & 2.12 & 1.25 & 0.51 & 0.0145 & 0.0144 & 0.037 & 0.1575 & bal. \\
\hline High O & 15.72 & 10.22 & 2.12 & 1.23 & 0.62 & 0.0148 & 0.0142 & 0.035 & 0.4814 & bal. \\
\hline \multirow{2}{*}{\multicolumn{6}{|c|}{ The Upper Limit of the Oxygen in the Commercial 316L Stainless Steel Powder }} & \multirow{2}{*}{\multicolumn{3}{|c|}{ carpenter additive }} & 0.10 & 22 \\
\hline & & & & & & & & & 0.10 & 23 \\
\hline
\end{tabular}
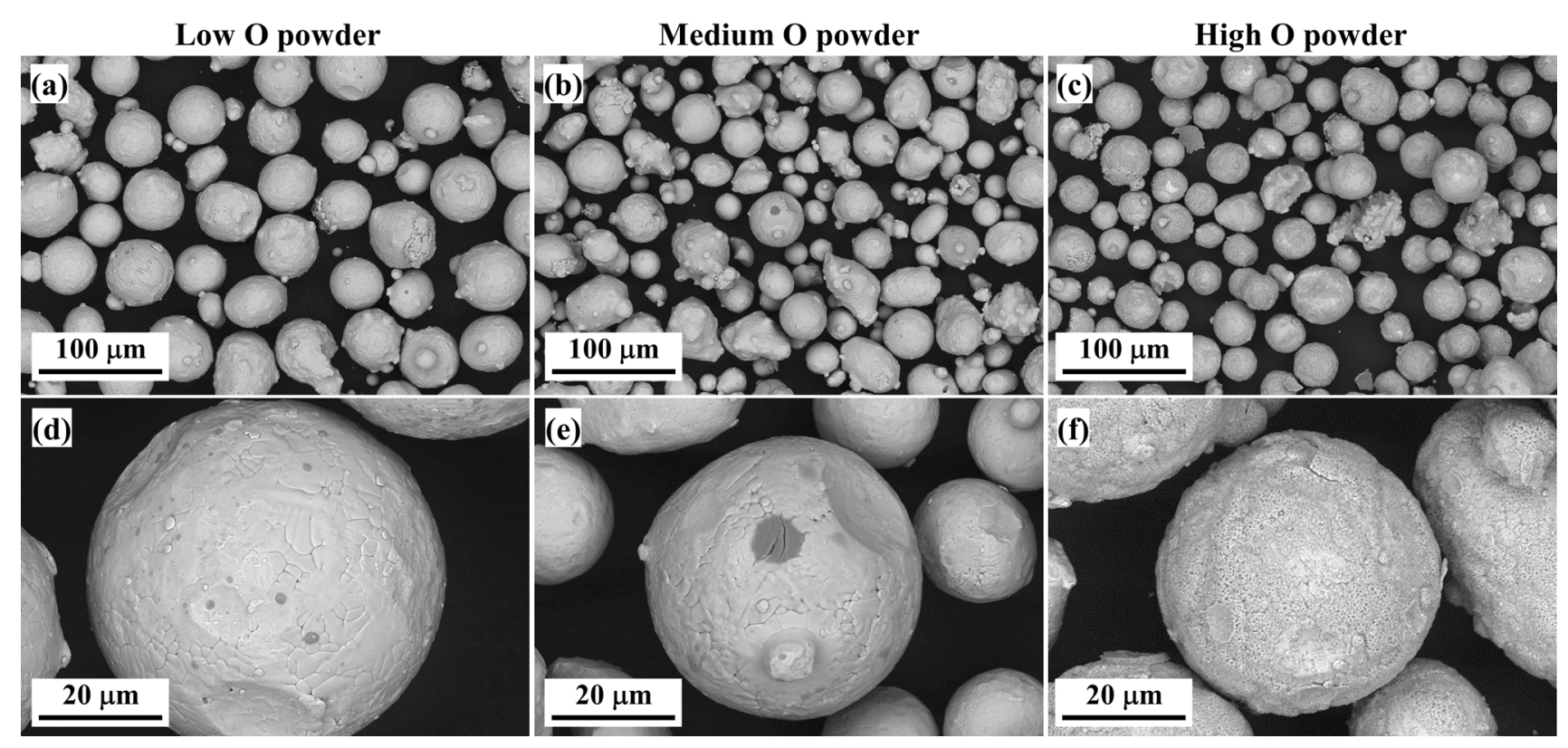

Fig. 1-Morphology of the three 316L powders with different oxygen pick-up conditions, $(a)$ and $(d)$ low $\mathrm{O}$ powder, $(b)$ and $(e)$ medium $\mathrm{O}$ powder, and $(c)$ and $(f)$ high $O$ powder.

\section{Non-equilibrium Thermodynamic Calculation}

The thermodynamic calculation was processed with FactSage 7.3 software package (GTT-Technologies). The Scheil-Gulliver cooling calculation was processed for the oxide formation in the non-equilibrium solidification $^{[31]}$ of $316 \mathrm{~L}$ stainless steel from its powder form with different oxygen pick-up conditions. The Scheil-Gulliver model ${ }^{[32]}$ assumes infinitely diffusion in the liquid phase, no diffusion in the solid phase, and local equilibrium at the solid/liquid interface. The gas-liquid and gas-solid reactions were excluded in the calculation due to the limited duration of the solidification process. Due to the limited mobility data of the element diffusion in oxide materials, the back diffusion from the solidified phase is not considered in the current calculation. As a reference, the equilibrium cooling calculation was also conducted to compare the oxide species prediction. The input data included the powder chemical composition and the different oxygen content for the given conditions. The database sets of FactPS, FToxid and FSstel were used. The slag liquid, monoxide, spinel, corundum, and rhodonite were selected as oxide solution candidates. The liquid, fcc austenite and bcc ferrite were selected as steel solution candidates.

\section{RESULTS AND DISCUSSION}

The experimental characterization and thermodynamic analysis are detailed in the following sections to reveal the oxide evolution of the droplet samples solidified from $316 \mathrm{~L}$ powders with different oxygen pick-up conditions.

\section{A. Oxide Evolution During the Solidification of $316 \mathrm{~L}$ Stainless Steel}

The snapshots of the in situ observation of the powder melting and solidification in HT-CLSM are presented in Figure 2. For the case of the low $\mathrm{O}$ powder, the gas bubble floating was observed from the molten liquid (Figure 2(a)) and then a clean melt surface was maintained (Figure 2(b)). The initiation of the dendritic structure was observed at $1437^{\circ} \mathrm{C}$ (Figure 2(c)). A fully developed dendrite could be seen in Figure 2(d) with decreasing temperature. The bubbling phenomenon suggested that the gas in between the powder materials was wrapped into the liquid pool in the melting stage, and then rapidly driven out of the melt pool by the buoyant force. In the case of the medium $\mathrm{O}$ powder, the oxides in the form of a porous network occurred on the 


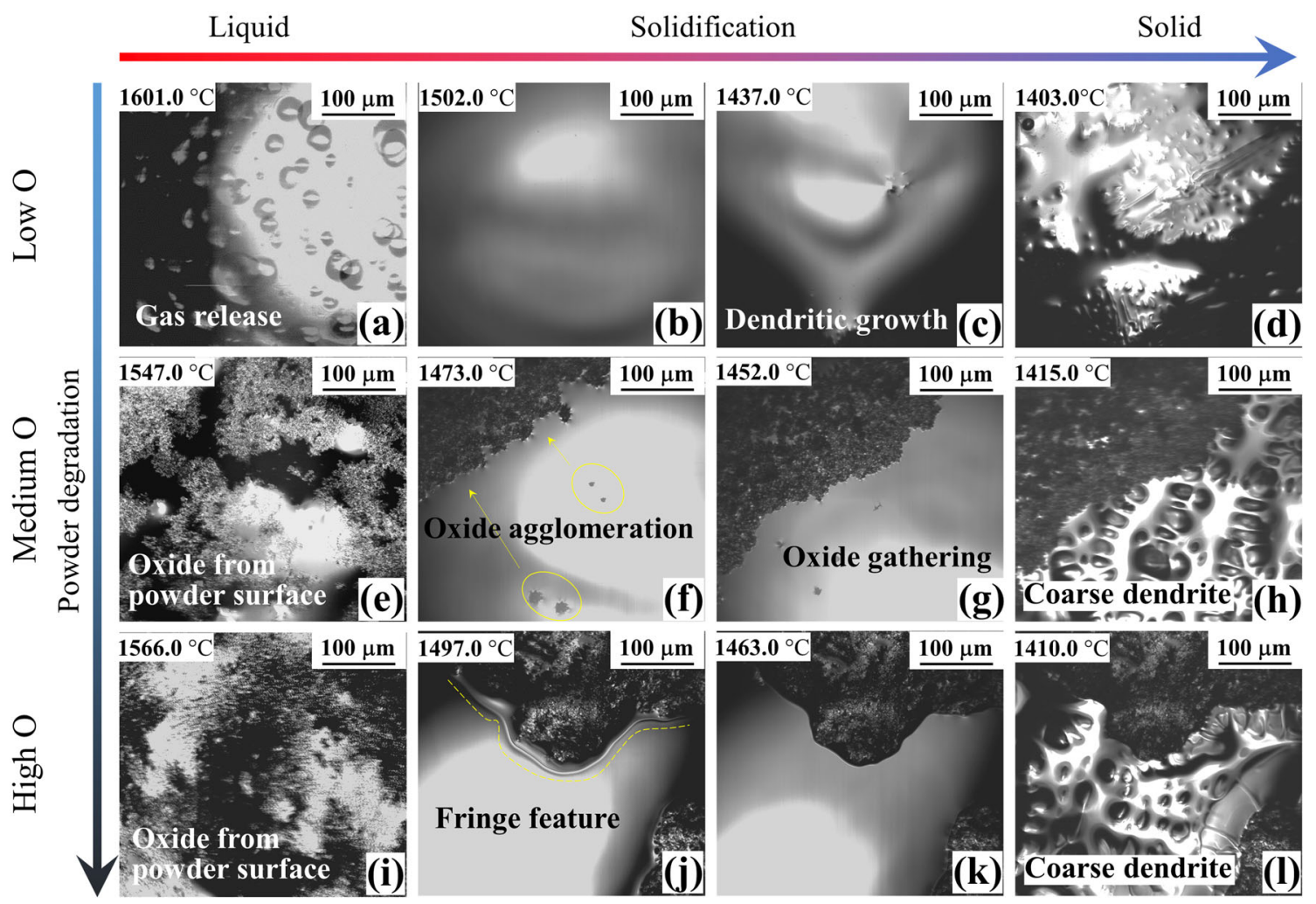

Fig. 2-The in situ observation of oxide evolution during the solidification of 316L stainless steel, $(a)$ through $(d)$ from low $\mathrm{O}$ powder, $(e)$ through $(h)$ from medium $\mathrm{O}$ powder, and $(i)$ through $(l)$ from high $\mathrm{O}$ powder.

top of the melt pool (Figure 2(e)) when the solid powders were transformed into an integrated liquid pool. With the assistance of interfacial energy and melt convection, the discrete oxide clusters were agglomerated as the oxide network. A significant oxide gathering was captured between Figures 2(f) and (g). The solidification of $316 \mathrm{~L}$ stainless steel from the high $\mathrm{O}$ powder in Figure 2(i) showed a similar oxide clustering phenomenon, but a thicker oxide layer compared to the medium $\mathrm{O}$ powder case. This is attributed to the high surface oxides formed in the powder oxidation treatment. During the floating up of the powder surface oxide with increased volume, the majority of the large oxide clusters has been integrated as a large oxide film shown on the top of the melt. A fringe feature was observed in Figure 2(j) between the melt and the oxide film at $1497^{\circ} \mathrm{C}$, which disappeared with further cooling (Figure 2(k)).

The observation in the HT-CLSM experiment shows that the powder oxygen pick-up due to the surface oxidation can influence the oxide evolution in the small volume powder fusion. Pre-existing surface oxide could release during the powder melting and gathered and agglomerated during solidification. Such agglomeration behavior assists the formation of continuous oxide films which can be entrapped in the as-processed component and potentially deteriorate the component mechanical performance. Eo et al. ${ }^{[12]}$ reported that the oxide layer covered the melt pool of $316 \mathrm{~L}$ stainless steel during the

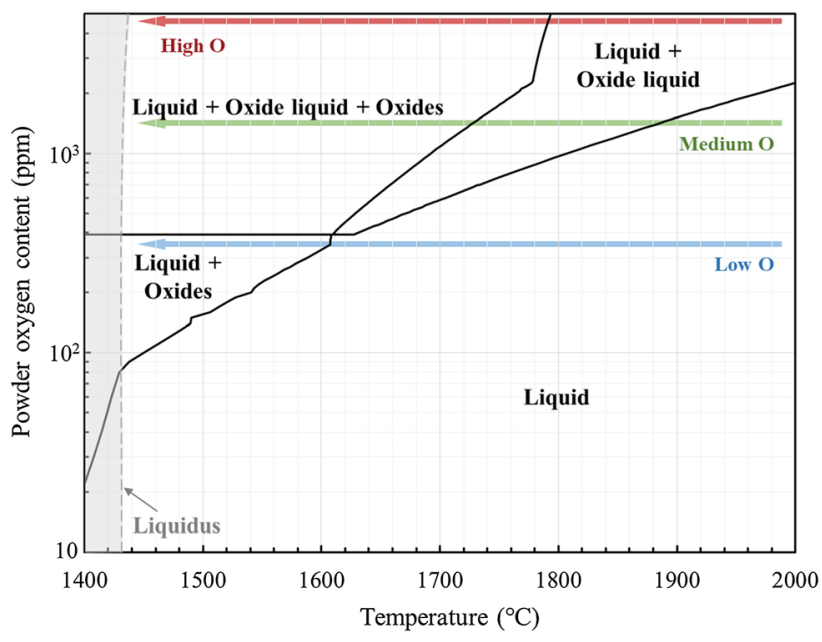

Fig. 3-The oxide evolution diagram of the 316L stainless steel with different oxygen content. The non-equilibrium calculation utilized the FactSage 7.3 and the Scheil-Gulliver cooling function. Liquid, liquid steel; Oxides, solid oxides; Oxide liquid, liquid Oxides.

laser powder deposition process. When a new layer of powders is deposited, the formed oxide layer can either melt or float up to the newly formed melt pool surface. Similar existence of the large oxide film was also reported in the Inconel 718 alloy. ${ }^{[33]}$ 
To understand the oxide evolution in the non-equilibrium solidification of $316 \mathrm{~L}$ stainless steel, an oxide evolution diagram was calculated using the Scheil-Gulliver cooling function in the FactSage 7.3 (Figure 3). The phase transition of the molten $316 \mathrm{~L}$ powder with different oxygen contents was calculated by the 10 ppm interval through the given temperature range. It is clear from Figure 3 that the oxide evolution path during the non-equilibrium solidification varies significantly with the oxygen content in the powder. For the oxygen content in the powder above $390 \mathrm{ppm}$ (i.e., medium $\mathrm{O}$ powder and high $\mathrm{O}$ powder), it tends to form a liquid oxide at high temperature (e.g., $1890{ }^{\circ} \mathrm{C}$ for medium $\mathrm{O}$ powder and above $2000{ }^{\circ} \mathrm{C}$ for high $\mathrm{O}$ powder) when the total oxygen content in the steel is more than the oxygen solubility of the corresponding temperature. During the non-equilibrium solidification (temperature decreasing), solid oxides form (slag crystallizes). This oxide evolution path promotes the agglomeration of the non-metallic phases in a wide temperature range due to the driving force of interfacial energy difference between immiscible steel melt and the oxide liquid, as well as the low surface tension of the crystallized (solid) oxide and the liquid oxide. One may suggest that the temperature-induced Marangoni convection may disperse the clustered oxide layer in the high energy beam-powder interaction. According to the oxide agglomeration model ${ }^{[34]}$ and the Marangoni flow characterization, ${ }^{[35]}$ the turbulence kinetic energy is $\sim 0.35 \mathrm{~m}^{2} / \mathrm{s}^{2}$ by assuming turbulence intensity is 50 pct of the mean flow rate, which is still lower than the energy required to disperse the oxides agglomerate smaller than $100 \mu \mathrm{m}\left(0.75 \mathrm{~m}^{2} / \mathrm{s}^{2}\right)$. The oxide agglomeration may well exist in the fusion process from the steel powder with excessive oxygen pick-up during its service life. It can either pre-exist or melt and solidify to form the oxide liquid and then crystallize.

For the oxygen content in the powder lower than 390 ppm (e.g., low O powder), oxide liquid is not formed during non-equilibrium solidification but solid oxides uniformly precipitate in the liquid steel under preferred thermal conditions. The contact of the neighboring oxide precipitates can be determined by the Brownian motion, the sedimentation by density difference and the internal convention. However, Brownian motion is a time-dependent phenomenon, ${ }^{[36]}$ and the drag force of the oxide sedimentation is limited by the particle size (micron to sub-micron). ${ }^{[37]}$ Therefore, limited clustering/agglomeration in powder fusion due to the un-favored kinetics condition is expected, which is consistent with the experimental observation of a clean melt surface in the solidification of $316 \mathrm{~L}$ stainless steel from low O powder.

The oxide formation and evolution path are of great importance in the control of the clustering/agglomeration defect. Ideally, the oxygen content of the $316 \mathrm{~L}$ powder material should be below $\sim 390 \mathrm{ppm}$ for the uniformly precipitated oxide particles in the steel melt. With the increasing oxygen content, the oxide agglomeration becomes severe as seen in the medium $\mathrm{O}$ case with 0.1575 wt pct oxygen and even worse in the high $\mathrm{O}$ powder with $0.4814 \mathrm{wt}$ pct oxygen content.

\section{B. Oxide Composition of the As-solidified 316L Stainless Steel Droplet}

The as-solidified droplets from the melting and solidification experiments of the three powders in HT-CLSM are taken to analyze the inherent influence of the powder oxygen pick-up on its oxide evolution during the solidification. Three distinctive morphologies of inclusions are exhibited on the surface of the as-solidified droplets from the three different powders. The droplet made by the low $\mathrm{O}$ powder shows a smooth surface curved by fine dendrite tips in Figures 4(a) and (d) and its detailed microstructure (Figure 4(g)). The Si-rich oxides (gray contrast) at the size of several microns and finer chromium-rich oxides (bright contrast) in sub-micron scale were identified by the EDS chemical analysis. According to Figure 3, the oxide precipitates directly in the steel melt during the solidification of the $316 \mathrm{~L}$ melt from the low $\mathrm{O}$ powder $(0.035$ wt pct $\mathrm{O}$ ). The formed oxide phase is predicted by FactSage calculation to be $(\mathrm{Mn}, \mathrm{Cr}) \mathrm{Cr}_{2} \mathrm{O}_{4}$ spinel at $1607.37^{\circ} \mathrm{C}$ and then $\mathrm{SiO}_{2}$ oxides.

Figures 4(b), (e), and (h) show the surface morphology of the droplet solidified from the melt of the medium $\mathrm{O}$ powder in the HT-CLSM experiment. The droplet sample is partially covered with the oxides observed in the Figures 2(e) through (h), and the steel surface presents a pattern of directional dendritic structure with severe shrinkage. The RoI 1 and RoI 2 are two representative oxide morphologies illustrated in Figure 4(b). The RoI 1 (Figure 4(e)) shows the silica particles and chromium oxides uniformly formed on the droplet surface similar to the case of the low $\mathrm{O}$ powder. The RoI 2 area (Figure 4(h)) corresponds to the porous oxide network observed during the melting and solidification, which was inherited from the medium $\mathrm{O}$ powder surface.

The TEM characterization of the FIB sliced oxide crystal is presented in Figure 5. The well-developed facet structure was observed in the High-angle annular dark-field (HAADF) image (Figure 5(a)) with the compounds entrapped within the oxide crystal. The selected area diffraction (SAD) pattern (Figure 5(b)) of the same crystal shows a cubic crystal structure with an incident beam parallel to the [111] zone axis. Together with the EDS mapping and quantitative analysis of Figures 5(d) through (g), the oxide observed in Figure 4(h) is confirmed to be $\mathrm{MnCr}_{2} \mathrm{O}_{4}$ spinel oxide. The entrapped compound indicated in Figure 5(a) is confirmed as amorphous silicon oxide by the brightfield (BF) image with the insertion of Fast Fourier transform (FFT) in Figure 5(c) and EDS mapping in Figures $5(\mathrm{~g})$ and $(\mathrm{h})$. An iron particle is also observed in the oxide crystal as shown in Figure 5(d), which could be the entrapped liquid steel in the oxide crystallization.

The thick and large patches of inclusions (oxides) are observed on the surface of the as-solidified droplet from the high O powder during the HT-CLSM experiment in Figure 4(c) and the two RoIs (Figures 4(f) and (i)). In RoI 1, three sections of the droplet surface have been separated by dash lines, which are the steel surface, 


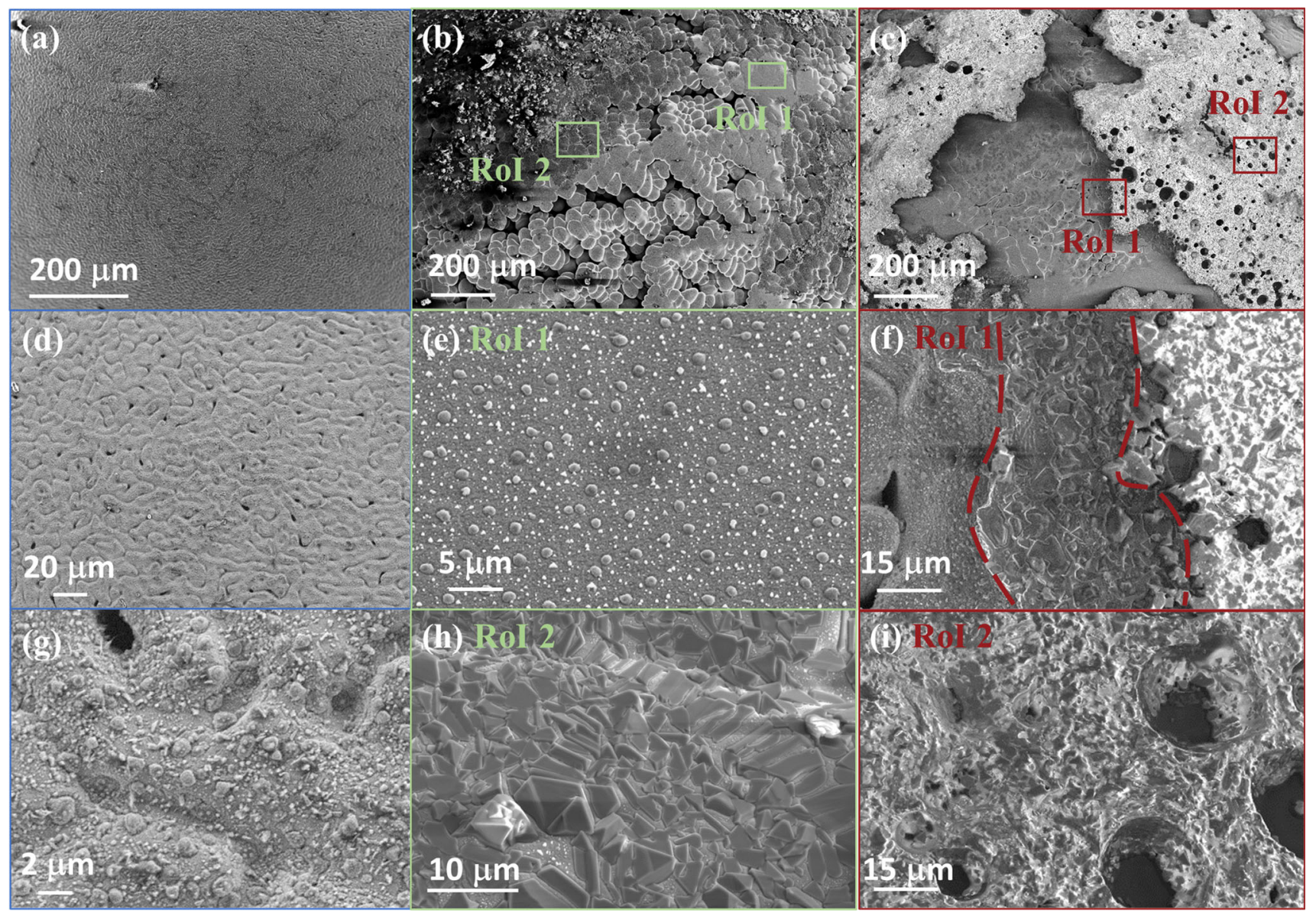

Fig. 4-The surface morphology of droplets solidified from different powder materials, $(a),(d)$, and $(g)$ low $\mathrm{O}$ powder, $(b),(e)$, and $(h)$ medium $\mathrm{O}$ powder, and $(c),(f)$, and $(i)$ high $\mathrm{O}$ powder. RoI, Region of Interest.

intermediate phase and the thick layer of oxides corresponding to the liquid metal, the fringe feature and the oxides layer in the HT-CSLM experiment (Figure 2(j)). The thick oxide layer in RoI 2 (Figure 4(i)) could be a mixture of silicon oxides and $\mathrm{Cr}$, Mn containing oxides from the EDS analysis. The silicon oxides present a bright contrast, while the dark contrast is the Cr, Mn-containing oxides. In Figures 6(a) and (b), the thick oxide layer in RoI 2 (Figure 4(i)) is confirmed to be a mixture of dark facet crystals and bright binder compound. The high-resolution TEM-BF images (Figures 6(c) and (d)) showed a cubic crystal structure with (111) plane $d$-spacing of $0.4873 \mathrm{~nm}$ for the dark facet crystal, while an amorphous feature for bright binder compound (Figure 6(e)). Together with the chemical analysis in Figures 6(g) through (j), the dark facet crystal is $\mathrm{MnCr}_{2} \mathrm{O}_{4}$ spinel ${ }^{[38]}$ and the bright compound is the amorphous silica. The intermediate phase observed in Figure 4(f) was analyzed using EDS through FIB sectioning due to its limited layer thickness (Figures 7(a) and (b)). The element mapping in Figures 7(e) through (h) suggests that the intermediate phase was mainly composed of a high fraction of the $\mathrm{Cr}$ and $\mathrm{Mn}$ containing oxide with a small volume of silicon oxides in between.
To understand the phase evolution in the oxide agglomeration on the medium $\mathrm{O}$ and high $\mathrm{O}$ droplets, the oxide phase calculations using the Scheil-Gulliver cooling and the equilibrium cooling functions are presented in Figure 8 by setting the oxide liquid as the cooling solution phase. In the equilibrium cooling, both cases follow the similar solidification route, i.e., Liquid (steel) + Oxide liquid $\rightarrow$ Spinel, by keeping the total mass balance constant. The temperature range of the above reaction is between $1633.71{ }^{\circ} \mathrm{C}$ to $1597.52{ }^{\circ} \mathrm{C}$ for the medium $\mathrm{O}$ melt, and $1697.56{ }^{\circ} \mathrm{C}$ to $1568.41{ }^{\circ} \mathrm{C}$ for the high $\mathrm{O}$ melt. However, once the solid phase has been removed from the total mass balance of the target phase in the Scheil-Gulliver cooling, the solidification routes significantly deviated from the equilibrium state. In the medium $\mathrm{O}$ case (Figure $8(\mathrm{a})$ ), the $(\mathrm{Mn}, \mathrm{Cr}) \mathrm{Cr}_{2} \mathrm{O}_{4}$ spinel first formed at $1736.26^{\circ} \mathrm{C}$ and consumed the majority of the oxide liquid, with further cooling, the $\mathrm{SiO}_{2}$ oxide occurred at $1482.98{ }^{\circ} \mathrm{C}$. This is consistent with the oxide phase identified in Figure 5. However, the $\mathrm{SiO}_{2}$ was presented in an amorphous state rather than the tridymite crystal structure due to the influence of the cooling rate subjected to the experiment. A good agreement is obtained between the oxide phase identified in the TEM characterization (Figure 6) and the 


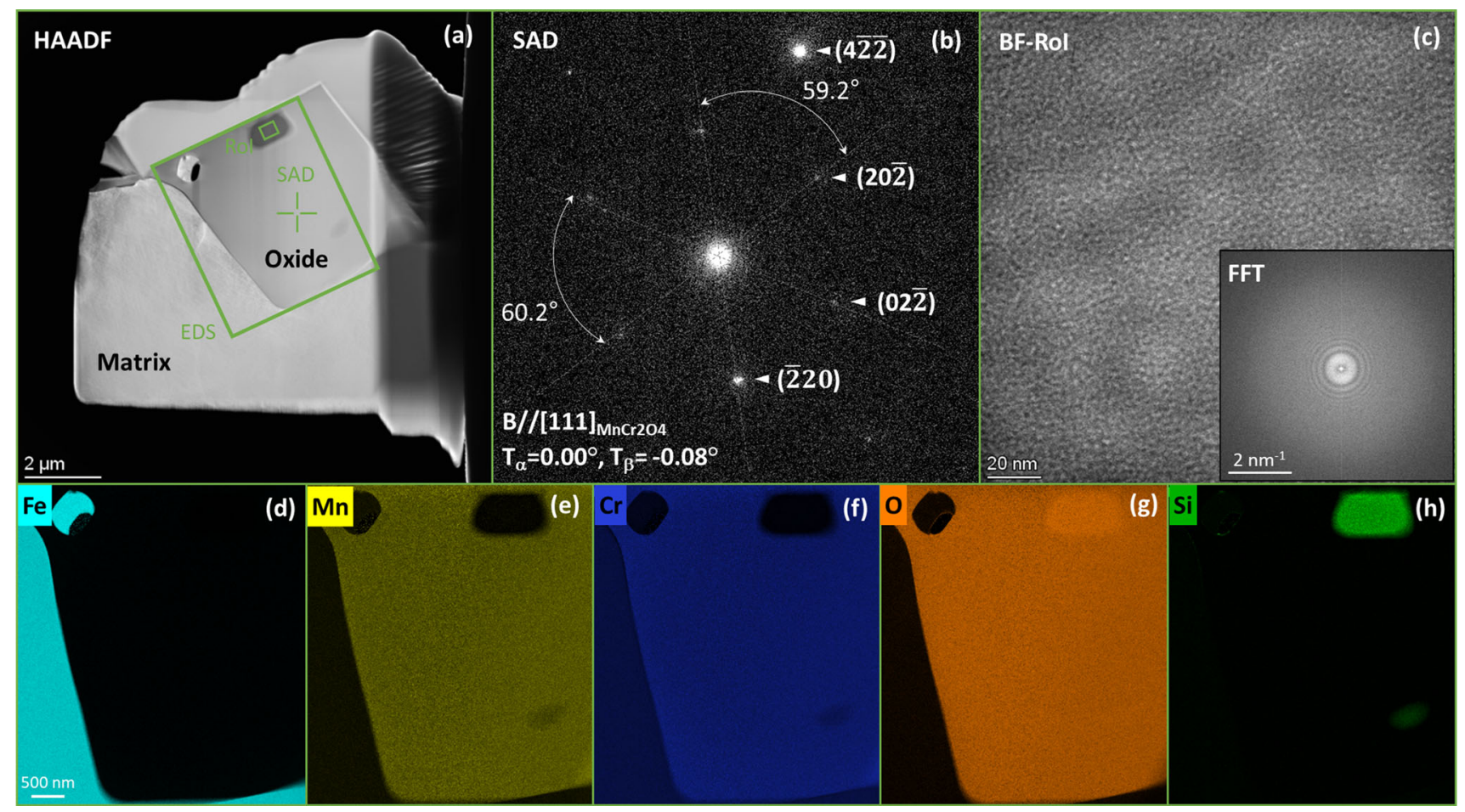

Fig. 5-The crystal structure identification and chemical analysis of the oxide shown in the RoI 2 in the medium O droplet (Fig. 4(h)), (a) HAADF image of the oxide crystal, $(b)$ selected area diffraction (SAD) pattern of the same oxide, $(c)$ the BF image of the entrapped compound $\mathrm{RoI}$ in (a) with the insertion of FFT image suggesting a feature of amorphous structure, and $(d)$ through $(h)$ the element maps of Fe, Mn, Cr, O, and $\mathrm{Si}$ to confirm the oxide from chemical analysis.

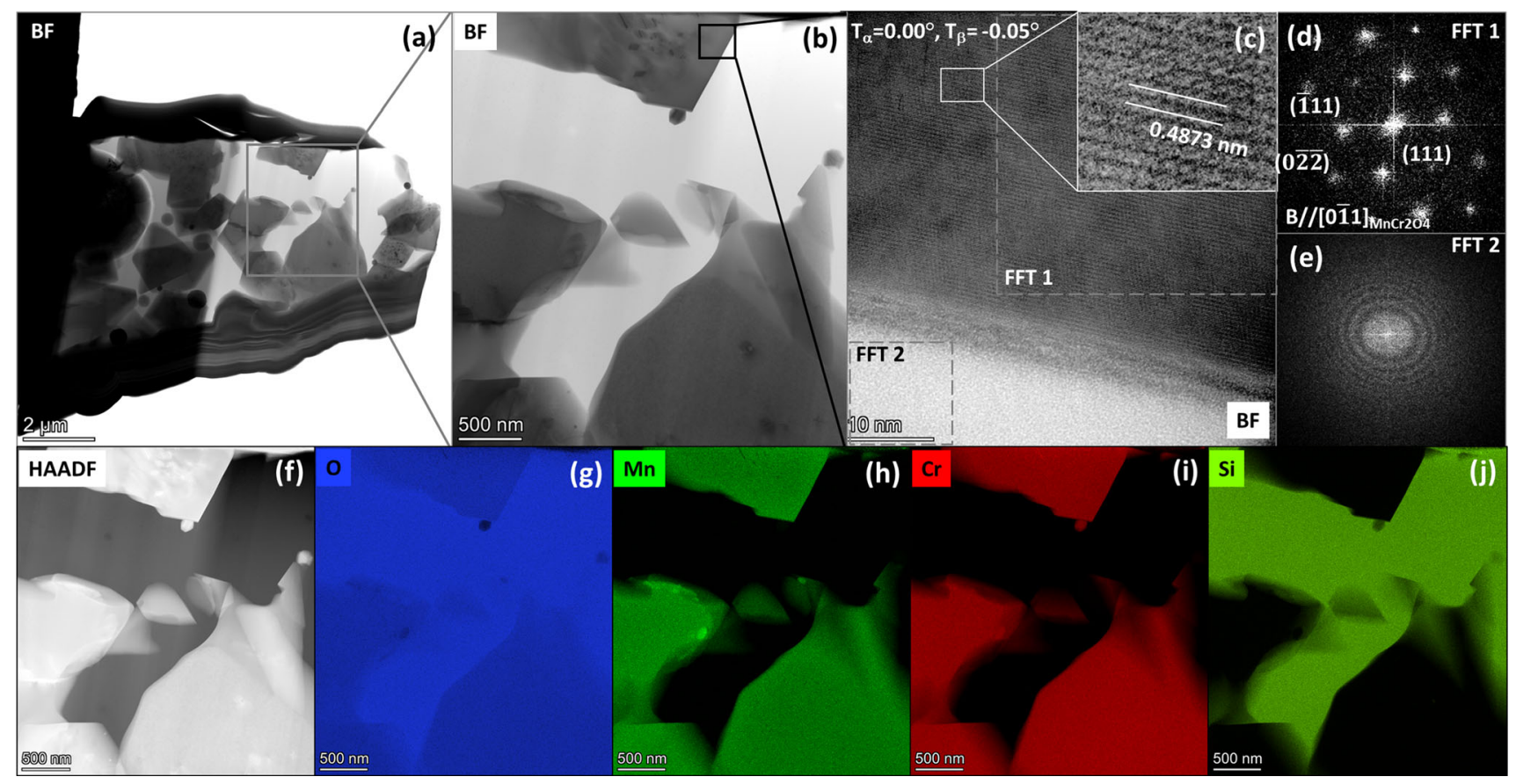

Fig. 6-The crystal structure identification and chemical analysis of the oxides shown in the RoI 2 in the high O droplet (Fig. 4(i)), (a) and (b) BF image of the oxide mixture, $(c)$ the high-resolution TEM with the two FFT images $(d)$ and $(e)$ of the selected area, $(f)$ the HAADF image of the mixture of the oxide layer, and $(g)$ through $(j)$ the element maps of $\mathrm{O}, \mathrm{Mn}, \mathrm{Cr}$, and $\mathrm{Si}$ to confirm the oxide from chemical analysis. 

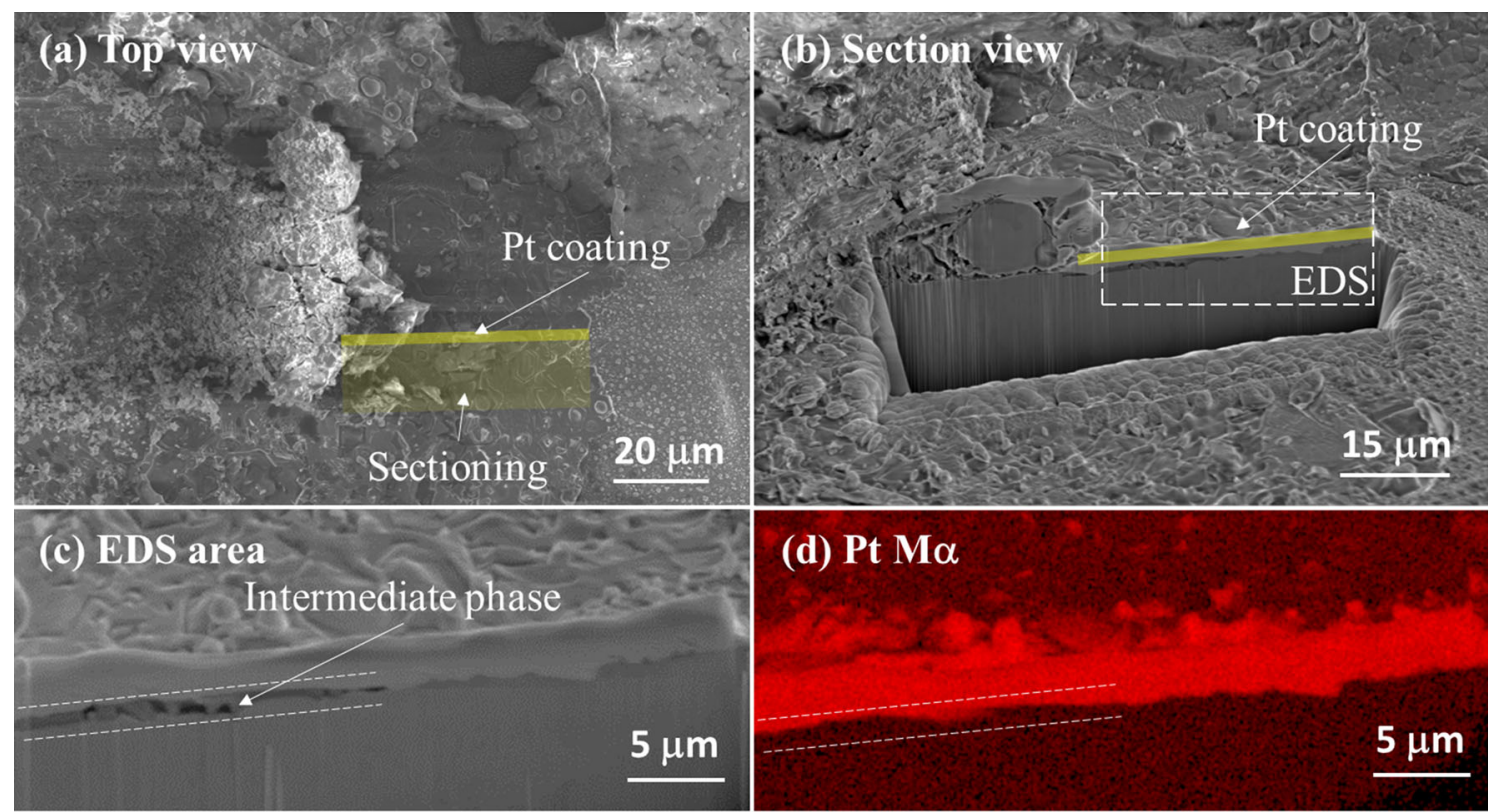

(d) Pt M $\alpha$
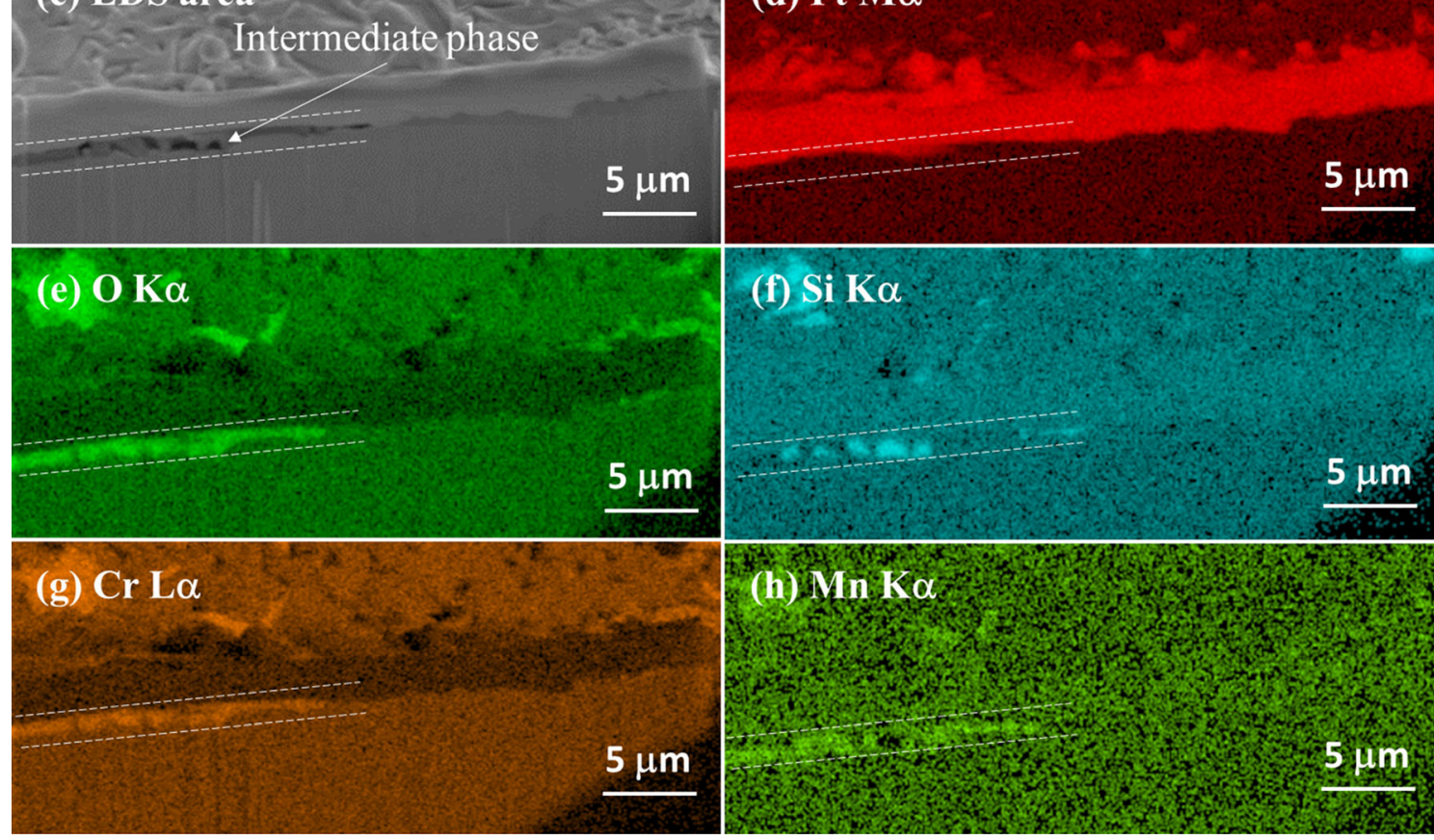

(f) Si Ka

Fig. 7-The EDS analysis of intermediate phase observed on the surface of the droplet solidified from the high O powder Fig. 4(f)) through the FIB sectioning, (a) the top view of intermediate phase before sectioning, $(b)$ the section view of the sectioned area with 52 deg tilting of stage $z$-axis, $(c)$ the selected EDS mapping area with the cross-section of the intermediate phase, and $(d)$ through $(h)$ the element mapping of platinum, oxygen, silicon, chromium, and manganese.

non-equilibrium thermodynamic analysis in Figure 8(b) for the high $\mathrm{O}$ powder case. The thermodynamic calculation suggests an even higher spinel formation temperature of $1792.68^{\circ} \mathrm{C}$ for spinel crystallization from the oxide liquid.

The non-equilibrium oxide evolution of the solidification of $316 \mathrm{~L}$ stainless steel from powder form with different oxygen contents has been investigated in this work by correlating the experimental results (in situ observation and advanced characterization) with the non-equilibrium thermodynamic analysis. A relationship between the oxide formation/evolution route and the powder oxygen content has been established and the resultant oxide species was predicted by utilizing the Scheil-Gulliver cooling function and verified through crystallographic and chemical identification.
The current work suggests that the oxide formation and the resultant species under the non-equilibrium condition deviated significantly from its equilibrium state in particular for the powders containing medium and high oxygen contents (>390 ppm). This may improve the oxide inclusion prediction in rapid solidification processes, such as the powder fusion-based additive manufacturing, where the inclusion engineering is lack of consideration at the moment, however, could play a critical role, due to its inherent high oxygen content in the metal powder, in the properties of the as-built components. For the low $\mathrm{O}$ powder, it favors the powder fusion-based AM process due to its much lower oxide formation temperature and eliminated liquid oxide phase in the non-equilibrium solidification, which leads to the fine inclusions precipitating uniformly in the 


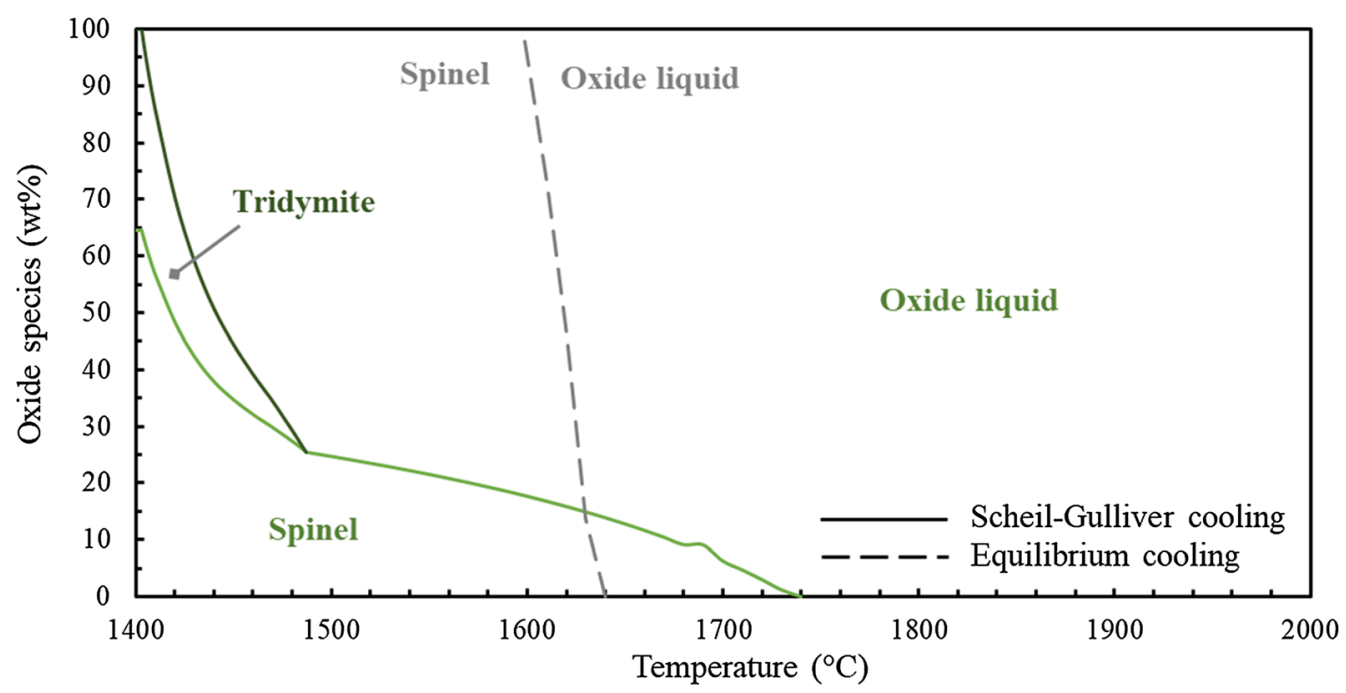

(a)

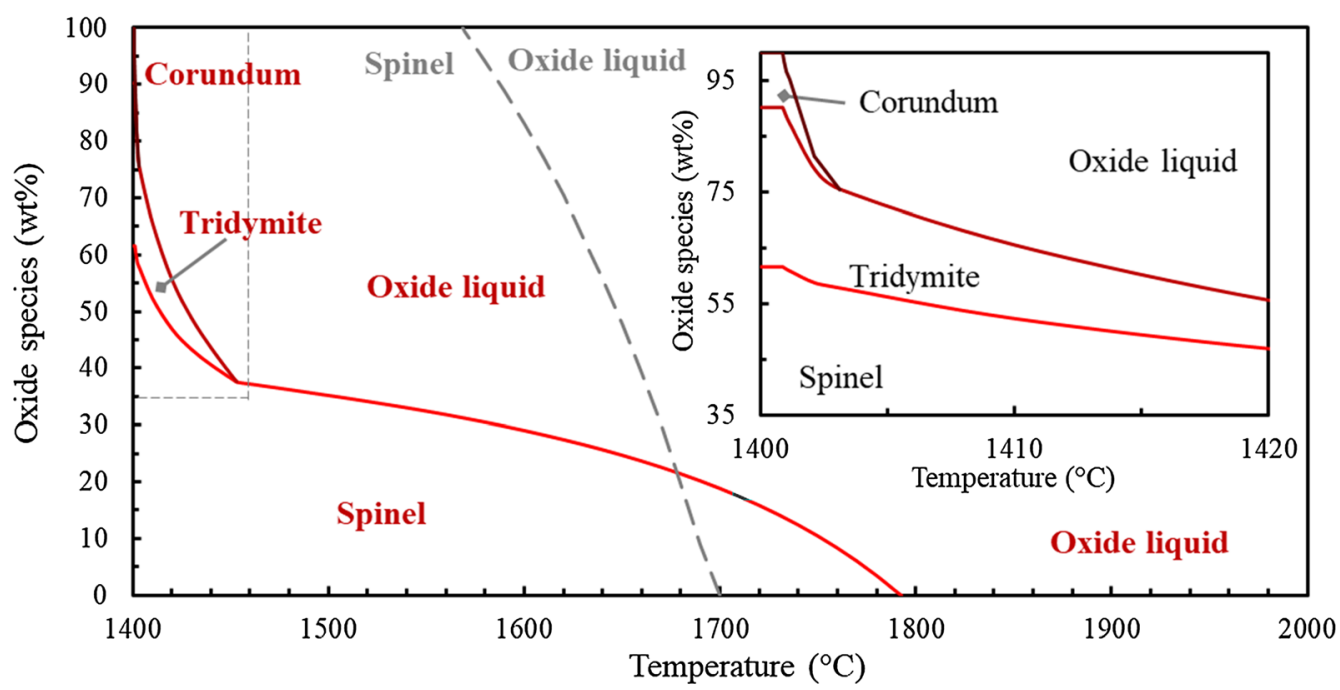

(b)

Fig. 8-The predicted oxide phase evolution as a function of the temperature through Scheil-Gulliver cooling (solid line) and equilibrium cooling (gray dashed line) calculation, $(a)$ medium $\mathrm{O}$ powder material, and $(b)$ high $\mathrm{O}$ powder material. Here, the spinel phase is composed by $(\mathrm{Mn}, \mathrm{Cr}) \mathrm{Cr}_{2} \mathrm{O}_{4}$, tridymite is $\mathrm{SiO}_{2}$, and corundum is $\mathrm{Cr}_{2} \mathrm{O}_{3}$.

as-built component. The medium and high $\mathrm{O}$ powders may not be suitable for the conventional AM component due to the excessive oxide inclusion. The unique feature of metallic powder coated with oxide layer may enable them into the functional grade materials, e.g., to improve the stiffness of the component made by retained oxide network in between the steel matrix uniformly.

\section{CONCLUSIONS}

In the current work, the oxide evolution in the solidification of $316 \mathrm{~L}$ stainless steel from additive manufacturing powders with different oxygen contents was investigated through the in situ observation, TEM phase identification, and non-equilibrium thermodynamic analysis. The conclusions can be drawn below.
1. The oxygen pick-up on the powder materials through surface oxidation significantly influences the oxide formation/evolution, morphology, and species during the solidification of the $316 \mathrm{~L}$ stainless steel from its powder form.

2. The Scheil-Gulliver cooling-based non-equilibrium thermodynamic calculation of the oxide evolution provides consistent oxide phase prediction to the crystallographic phase identification, and it deviates from the equilibrium cooling function.

3. For the powder with oxygen content higher than $390 \mathrm{ppm}$ (medium and high O powder), the formation of liquid oxide mixture from the steel melt and then crystallization to certain oxide phases are observed during melting and solidification, while for the powder with lower than $\sim 390 \mathrm{ppm}$ oxygen (low O powder), such oxide evolution behavior is not ob- 
served and oxide phases are suggested to form from the steel melt uniformly during solidification.

4. The oxides formed in the melt of the low O 316L alloy $(0.0355$ pct $\mathrm{O})$ are predicted to be $(\mathrm{Mn}$, Cr) $\mathrm{Cr}_{2} \mathrm{O}_{4}$ spinel and $\mathrm{SiO}_{2}$ oxide. With increasing oxygen content, oxide liquid forms first, and then the spinel phase crystallizes from the oxide liquid at the temperature of $1736.26^{\circ} \mathrm{C}$ and $\mathrm{SiO}_{2}$ at $1482.98{ }^{\circ} \mathrm{C}$ in the medium $\mathrm{O}$ case $(0.1575$ pct $\mathrm{O})$. In the high $\mathrm{O}$ $(0.4814$ pct $\mathrm{O}) 316 \mathrm{~L}$ melt solidification, the formation temperature of spinel is raised to $1792.68^{\circ} \mathrm{C}$. The final oxides formed are $(\mathrm{Mn}, \mathrm{Cr}) \mathrm{Cr}_{2} \mathrm{O}_{4}$ spinel, $\mathrm{SiO}_{2}$ oxide, and $\mathrm{Cr}_{2} \mathrm{O}_{3}$ corundum.

\section{ACKNOWLEDGMENTS}

This work is financially supported by EPSRC with Grant No. EP/N011368/1. The authors would like to thank Dr. Sabrina Yan for the TEM sample preparation in WMG, the University of Warwick.

\section{OPEN ACCESS}

This article is licensed under a Creative Commons Attribution 4.0 International License, which permits use, sharing, adaptation, distribution and reproduction in any medium or format, as long as you give appropriate credit to the original author(s) and the source, provide a link to the Creative Commons licence, and indicate if changes were made. The images or other third party material in this article are included in the article's Creative Commons licence, unless indicated otherwise in a credit line to the material. If material is not included in the article's Creative Commons licence and your intended use is not permitted by statutory regulation or exceeds the permitted use, you will need to obtain permission directly from the copyright holder. To view a copy of this licence, visit http://creativec ommons.org/licenses/by/4.0/.

\section{REFERENCES}

1. J.F. Elliott and M. Gleiser: Thermochemistry for Steelmaking, Addison-Wesley Publishing Co., Reading, 1963.

2. D. Herzog, V. Seyda, E. Wycisk, and C. Emmelmann: Acta Mater., 2016, vol. 117, pp. 371-92.

3. J. Oliveira, T. Santos, and R. Miranda: Prog. Mater. Sci., 2020, vol. 107 , p. 100590.

4. T. DebRoy, T. Mukherjee, J. Milewski, J. Elmer, B. Ribic, J. Blecher, and W. Zhang: Nat. Mater., 2019, vol. 18, pp. 1026-32.

5. S. Öztürk, K. İçin, B. Öztürk, U. Topal, and H.K. Odabasi: Int. J. Mater. Sci. Appl., 2017, vol. 6, pp. 241-49.

6. S. Öztürk, S.E. Sünbül, and K. İcin: Trans. Nonferr. Met. Soc. China, 2020, vol. 30, pp. 1169-82.
7. T. Lau, G. Weatherly, and A. McLean: Weld. J., 1986, vol. 65, pp. 343-47.

8. T. Kuwana, Y. Sato, and S. Kaneda: Weld. Int., 1993, vol. 7, pp. $365-70$.

9. T. Koseki, S. Ohkita, and N. Yurioka: Sci. Technol. Weld. Join., 1997, vol. 2, pp. 65-69.

10. O. Grong, T. Siewert, G. Martins, and D. Olson: Metall. Mater. Trans. A, 1986, vol. 17A, pp. 1797-1807.

11. M. Song, X. Lin, F. Liu, H. Yang, and W. Huang: Mater. Des., 2016, vol. 90, pp. 459-67.

12. D. Eo, S. Park, and J. Cho: Mater. Des., 2018, vol. 155, pp. $212-$ 19.

13. P. Deng, M. Karadge, R.B. Rebak, V.K. Gupta, B.C. Prorok, and X. Lou: Addit. Manuf., 2020, vol. 35, p. 101334.

14. I.E. Anderson, E.M. White, and R. Dehoff: Curr. Opin. Solid State Mater. Sci., 2018, vol. 22, pp. 8-15.

15. E. Hryha, R. Shvab, H. Gruber, A. Leicht, and L. Nyborg: Metall. Ital., 2018, vol. 3, pp. 34-39.

16. S.E. Brika, M. Letenneur, C.A. Dion, and V. Brailovski: Addit. Manuf., 2020, vol. 31, p. 100929.

17. A.B. Spierings, M. Voegtlin, T. Bauer, and K. Wegener: Prog. Addit. Manuf., 2016, vol. 1, pp. 9-20.

18. P. Quinn, S. O'Halloran, J. Lawlor, and R. Raghavendra: $A d v$. Mater. Process. Technol., 2019, vol. 5, pp. 348-59.

19. Z. Dong, H. Kang, Y. Xie, and C. Chi: Mater. Lett., 2019, vol. 236, pp. 214-17.

20. M.J. Heiden, L.A. Deibler, J.M. Rodelas, J.R. Koepke, D.J. Tung, and D.J. Saiz: Addit. Manuf., 2019, vol. 25, pp. 84-103.

21. C.L.A. Leung, S. Marussi, M. Towrie, R.C. Atwood, P.J. Withers, and P.D. Lee: Acta Mater., 2019, vol. 166, pp. 294-305.

22. Carpenter Additive technical data sheet CT PowderRange 316L F. https://www.carpenteradditive.com/hubfs/carpenter_additive/im age/Resources/Datasheets/CT\%20PowderRange \%20316L\%20F. pdf. Accessed 10 Nov 2020.

23. Renishaw data sheet SS 316L-0407 powder for additive manufacturing. https://www.renishaw.com/media/pdf/en/f8cba72a8434 40d3bd8a09fd5021ad89.pdf. Accessed 10 Nov 2020.

24. S. Öztürk, S.E. Sünbül, A. Metoğlu, S. Önal, and K. İçin: Mater. Sci. Technol., 2020, vol. 36, pp. 1771-84.

25. H. Karlsson, L. Nyborg, and S. Berg: Powder Metall., 2005, vol. 48 , pp. $51-58$.

26. I. Nyborg, T. Tunberg, and P. Wang: Met. Powder Rep., 1990, vol. 45 , pp. $750-53$.

27. S.E. Sünbül, K. İçin, F.Z. Seren, Ö. Sahin, D.D. Çakil, R. Sezer, and S. Öztürk: Vacuum, 2021, vol. 187, p. 110072.

28. A. Erdogan, S.E. Sünbül, K. İçin, and K.M. Doleker: Vacuum, 2021, vol. 187, p. 110143

29. J. Zhou, H. Tsai, and P. Wang: Weld. J. Heat Transf., 2005, vol. 128 , pp. $680-90$.

30. C. Zhao, C. Kwakernaak, Y. Pan, I. Richardson, Z. Saldi, and S. Kenjeres: Acta Mater., 2010, vol. 58, pp. 6345-57.

31. D. Durinck, P.T. Jones, B. Blanpain, P. Wollants, G. Mertens, and J. Elsen: J. Am. Ceram. Soc., 2007, vol. 90, pp. 1177-85.

32. A.D. Pelton, G. Eriksson, and C.W. Bale: Metall. Mater. Trans. A, 2017, vol. 48A, pp. 3113-29.

33. Y. Zhang, X. Cao, P. Wanjara, and M. Medraj: Acta Mater., 2013, vol. 61 , pp. 6562-76.

34. H. Duan, Y. Ren, B.G. Thomas, and L. Zhang: Metall. Mater. Trans. B, 2019, vol. 50B, pp. 36-41.

35. S. Clark, C. Leung, Y. Chen, L. Sinclair, S. Marussi, and P. Lee: IOP Conf. Ser. Mater. Sci. Eng., 2020, vol. 861, p. 012010.

36. P. Shima, J. Philip, and B. Raj: Appl. Phys. Lett., 2009, vol. 94, p. 223101.

37. J Bellot, V. Descotes, and A. Jardy: JOM, 2013, vol. 65, pp. 1164 72.

38. J. Hastings and L. Corliss: Phys. Rev., 1962, vol. 126, p. 556.

Publisher's Note Springer Nature remains neutral with regard to jurisdictional claims in published maps and institutional affiliations. 